Marek Balcerzak and Wojciech Wojdowski, Institute of Mathematics, Łódź

Technical University, al. Politechniki 11, I-2, 90-924 Łódź, Poland,

e-mail: mbalce@@plunlo51.bitnet

\title{
SOME PROPERTIES OF ( $\Phi)$-UNIFORMLY SYMMETRICALLY POROUS SETS
}

\begin{abstract}
We prove that each perfect linear set contains a perfect set which is $(\Phi)$-uniformly symmetrically porous (Theorem 1). In the hyperspace of all nonempty compact sets in $[0,1]$ (endowed with the Hausdorff distance), the $(\Phi)$-uniformly symmetrically porous nonempty compact sets form a $G_{\delta}$ residual subspace (Theorem 2). We infer that the $(\Phi)$-uniformly symmetrically porous perfect sets form a $G_{\delta}$ residual set in the space of all perfect sets in $[0,1]$ (Theorem 3).
\end{abstract}

Each continuous increasing function $\Phi$ from $[0,1]$ into $[0,1]$ and fulfilling $\Phi(0)=0$ is called a porosity index. The set of all porosity indices will be denoted by $G$. The notion of $(\Phi)$-uniformly symmetric porosity, introduced in $[\mathrm{H}]$, constitutes (for the respective $\Phi \in G$ ) a sharper version of bilaterally strong porosity and strong symmetric porosity (cf. [H, Th. 3]). Our Theorems 1 and 2 describe the behaviour of $(\Phi)$-uniformly symmetrically porous sets in connection with the families of perfect sets and compact sets. That generalizes results known earlier for other kinds of porosity. Theorems 1 and 2 are used in the proof of Theorem 3 which states that a typical perfect set in $[0,1]$ is $(\Phi)$-uniformly symmetrically porous.

From now on, fix an arbitrary $\Phi \in G$. Let $n \in \mathbb{N}$. Following $[\mathrm{H}]$, we denote by $R(\Phi, n)$ the set of all $E \subseteq \mathbb{R}$ for which there are numbers $a_{n}, b_{n}$ such that for all $x \in E$ we have:

(i) $0<a_{n}<b_{n}<1 / n$,

(ii) $\Phi\left(b_{n}-a_{n}\right)>a_{n}$

(iii) $\left[x-b_{n}, x-a_{n}\right] \cap E=\left[x+a_{n}, x+b_{n}\right] \cap E=\emptyset$.

Key Words: porous set, perfect set, residual set, Hausdorff metric

Mathematical Reviews subject classification: Primary: 26A21, 54E52, 54B20, 04A15

Received by the editors December 29, 1994 
Let $R(\Phi)=\bigcap_{n=1}^{\infty} R(\Phi, n)$. Each set $E \in R(\Phi)$ is called $(\Phi)$-uniformly symmetrically porous.

The definitions of other kinds of porosity can be found in [Z]. For instance, if $E \subseteq \mathbb{R}, x \in \mathbb{R}$ and $r>0$ are fixed, denote by $l(E, x, r)$ the length of the longest interval $(a, b)$ such that $0<a<b<r$ and

$$
(x-b, x-a) \cap E=(x+a, x+b) \cap E=\emptyset .
$$

Then $E$ is called strongly symmetrically porous if

$$
\limsup _{r \rightarrow 0+} l(E, x, r) / r=1
$$

for each $x \in E$. Observe that, if $\Phi(x)=x^{2}$, then, by (i), (ii), we get

$$
a_{n} /\left(b_{n}-a_{n}\right)<b_{n}-a_{n}<b_{n}<1 / n .
$$

Hence $\lim _{n \rightarrow \infty} a_{n} /\left(b_{n}-a_{n}\right)=0$ or, equivalently $\lim _{n \rightarrow \infty}\left(b_{n}-a_{n}\right) / b_{n}=1$. This, by (iii), means that $(\Phi)$-uniform symmetric porosity implies strong symmetric porosity.

Theorem 1 Every linear perfect set contains a perfect $(\Phi)$-uniformly symmetrically porous set.

Proof. Fix any two decreasing sequences $\left\{x_{j}\right\}_{j=1}^{\infty}$ and $\left\{z_{j}\right\}_{j=1}^{\infty}$ of real numbers tending to zero. As $\Phi \in G$, the sequence $\left\{\Phi\left(z_{j}\right)\right\}_{j=1}^{\infty}$ is decreasing and it tends to zero. Choose a subsequence $\left\{x_{n_{j}}\right\}_{j=1}^{\infty}$ such that $x_{n_{j}}<\Phi\left(z_{j}\right)$ for every $j$. Put $y_{j}=x_{n_{j}}+z_{j}$ for $j \in \mathbb{N}$. Next pick a subsequence $\left\{y_{m_{j}}\right\}_{j=1}^{\infty}$ such that $y_{m_{j}}<1 / j$ for every $j$. Define $a_{j}=x_{n_{m_{j}}}$ and $b_{j}=y_{m_{j}}$ for $j \in \mathbb{N}$. Then for each $j \in \mathbb{N}$ we have $0<a_{j}<b_{j}<1 / j$ and $\Phi\left(b_{j}-a_{j}\right)>a_{j}$.

Now, let us turn to the main part of the proof. We may start with a perfect set $P \subseteq \mathbb{R}$ which is bounded. We will define by induction a family $\left\{P_{s}: s \in 2^{<\omega}\right\}$ of perfect subsets of $P$ where $2^{<\omega}$ stands for the set of all finite sequences of zeros and ones. If $s \in 2^{<\omega}$, we will denote $\min P_{s}=\alpha_{s}$, $\max P_{s}=\beta_{s}$ and $h_{n}=\min \left\{\beta_{s}-\alpha_{s}: s \in 2^{<\omega}, \operatorname{lh} s=n\right\}$ for $n \in \mathbb{N} \cup\{0\}$ where lh $s$ is the length of $s$. Let $P_{\langle\rangle}=P$ and fix $n \geq 0$. Assume that perfect sets $P_{s} \subseteq P$ are defined for all $s \in 2^{<\omega}$ with $\operatorname{lh} s<n$. We shall define the sets $P_{s \frown 0}$ and $P_{s \frown 1}$ for $\operatorname{lh} s=n$ (where $s \frown i$ is the respective extension of the sequence $s$ ). Let $k_{n}$ be the least positive integer satisfying $b_{k_{n}}+2 a_{k_{n}}<$ $h_{n}$. Choose $c_{s}$ (respectively, $d_{s}$ ) being a left-hand (respectively, right-hand) condensation point of $P_{s} \cap\left(\alpha_{s}, \alpha_{s}+a_{k_{n}}\right)$ (respectively, $P_{s} \cap\left(\beta_{s}-a_{k_{n}}, \beta_{s}\right)$ ). Put 
$P_{s \frown 0}=P_{s} \cap\left[\alpha_{s}, c_{s}\right]$ and $P_{s \frown 1}=P_{s} \cap\left[d_{s}, \beta_{s}\right]$ for all $s$ with lh $s=n$. Observe that for $t=s \frown i$ and $i \in\{0,1\}$, we have

$$
\beta_{t}-\alpha_{t}<a_{k_{n}}
$$

and

$$
\alpha_{s \frown 1}-\beta_{s \frown 0} \geq \beta_{s}-\alpha_{s}-2 a_{k_{n}} \geq h_{n}-2 a_{k_{n}}>b_{k_{n}}+2 a_{k_{n}}-2 a_{k_{n}}=b_{k_{n}} .
$$

Having all sets $P_{s}, s \in 2^{<\omega}$, we define

$$
Q=\bigcap_{n=1}^{\infty} Q_{n}
$$

where $Q_{n}=\bigcup\left\{P_{s}: s \in 2^{<\omega}, \operatorname{lh} s=n\right\}$. Then $Q$ is a perfect subset of $P$. Now we will show that the sequences $\left\{a_{k_{n}}\right\}_{n=1}^{\infty}$ and $\left\{b_{k_{n}}\right\}_{n=1}^{\infty}$ witness that $Q \in R(\Phi)$. For each $n \in \mathbb{N}$, conditions (i) and (ii) are clear, by the choice of the numbers $a_{k}$ and $b_{k}$. It suffices to prove (iii). Fix $n \in \mathbb{N}$ and $x \in Q$. Condition (iii) will be true, if

(iii') $\left[x-b_{k_{n}}, x-a_{k_{n}}\right] \cap Q_{n+1}=\left[x+a_{k_{n}}, x+b_{k_{n}}\right] \cap Q_{n+1}=\emptyset$.

Since $x \in Q$, therefore $x \in Q_{n+1}$ and thus $x \in P_{t}$ for some $t \in 2^{<\omega}, \operatorname{lh} t=n+1$. Now, (iii') can be derived from the following inequalities (compare (1) and (2)):

$$
\begin{aligned}
& x-a_{k_{n}} \leq \beta_{t}-a_{k_{n}}<\alpha_{t}, \\
& x+a_{k_{n}} \geq \alpha_{t}+a_{k_{n}}>\beta_{t},
\end{aligned}
$$

(it means that the intervals $\left[x-b_{k_{n}}, x-a_{k_{n}}\right]$ and $\left[x+a_{k_{n}}, x+b_{k_{n}}\right]$ do not meet $P_{t}$ ),

$$
\begin{aligned}
\left(b_{k_{n}}-a_{k_{n}}\right)+\alpha_{t}-\left(x-a_{k_{n}}\right) & \leq b_{k_{n}}-a_{k_{n}}+a_{k_{n}}=b_{k_{n}}, \\
\left(b_{k_{n}}-a_{k_{n}}\right)+x+a_{k_{n}}-\beta_{t} & \leq b_{k_{n}}-a_{k_{n}}+a_{k_{n}}=b_{k_{n}},
\end{aligned}
$$

(it means that the intervals $\left[x-b_{k_{n}}, x-a_{k_{n}}\right]$ and $\left[x+a_{k_{n}}, x+b_{k_{n}}\right]$ do not meet the set $P_{s} \subseteq Q_{n+1}$ closest to $\left.P_{t}\right)$.

Note that the results analogous to Theorem 1 for strong symmetric porosity in $\mathbb{R}$ and for strong porosity in a Polish space were proved in [B, Th. 1.4, 1.6].

Let $\mathbb{K}$ denote the space of all nonempty compact sets in $[0,1]$ endowed with the Hausdorff metric $\rho$ given by

$$
\rho(H, F)=\max \left\{\sup _{x \in H} d(x, F), \sup _{y \in F} d(y, H)\right\}
$$

where $d$ is the usual metric on $[0,1]$. It is known that $\mathbb{K}$ forms a Polish space $[\mathrm{K}]$. 
Theorem 2 The $(\Phi)$-uniformly symmetrically porous nonempty compact sets in $[0,1]$ form a $G_{\delta}$ dense subset of $\mathbb{K}$, and therefore it is a residual set in $\mathbb{K}$.

Proof. Since the nonempty finite sets form a dense set (in $\mathbb{K}$ ) which is a subset of $R(\Phi)$, it suffices to show that $R(\Phi, n) \cap \mathbb{K}$ is open for each $n \in \mathbb{N}$. So, let $n \in \mathbb{N}$ and let $F \in R(\Phi, n) \cap \mathbb{K}$. Assume that numbers $a_{n}$ and $b_{n}$ fulfill (i),(ii) and (iii). Put

$$
U=\bigcup_{x \in F}\left(\left(x-b_{n}, x-a_{n}\right) \cup\left(x+a_{n}, x+b_{n}\right)\right) .
$$

Then $U$ is open and bounded. We can express $U$ as $\bigcup_{j}\left(c_{j}, d_{j}\right)$ where the intervals $\left(c_{j}, d_{j}\right)$ are pairwise disjoint. Since $d_{j}-c_{j} \geq b_{n}-a_{n}$ for every $j$, therefore $U=\bigcup_{j=1}^{p}\left(c_{j}, d_{j}\right)$ for some $p \in \mathbb{N}$. Observe that $c_{j} \notin F$ and $d_{j} \notin F$ for $j=1, \ldots, p$. For instance, we will show that $c_{j} \notin F$. If $c_{j}=x-b_{n}$ or $c_{j}=x+a_{n}$ for some $x \in F$, condition $c_{j} \notin F$ is clear by (iii). In the other case, there is a sequence $\left\{x_{m}\right\}_{m=1}^{\infty} \subseteq F$ such that

$$
c_{j}=\lim _{m \rightarrow \infty}\left(x_{m}-b_{n}\right) \text { or } c_{j}=\lim _{m \rightarrow \infty}\left(x_{m}+a_{n}\right) .
$$

By the compactness of $F$, choose a subsequence $x_{i_{m}}$ tending to $x \in F$. Thus $c_{j}=x-b_{n}$ or $c_{j}=x+a_{n}$ which again by (iii), yields that $c_{j} \notin F$. Define

$$
\varepsilon=(1 / 2) \min \left\{d\left(c_{j}, F\right), d\left(d_{j}, F\right): j=1, \ldots, p\right\} .
$$

Then the ball $B=\{H \in \mathbb{K}: \rho(H, F)<\varepsilon\}$ is contained in $R(\Phi, n) \cap \mathbb{K}$. Indeed, if $H \in B$ then $H \subseteq D$ where $D=\bigcup_{x \in F}(x-\varepsilon, x+\varepsilon)$. Consider any $y \in H$. Then $y \in(x-\varepsilon, x+\varepsilon)$ for some $x \in F$. Observe that

$$
\begin{aligned}
{\left[y-b_{n}, y-a_{n}\right] \cup\left[y+a_{n}, y+b_{n}\right] } & \subseteq\left[x-\varepsilon-b_{n}, x+\varepsilon-a_{n}\right] \cup\left[x-\varepsilon+a_{n}, x+\varepsilon+b_{n}\right] \\
& \subseteq \bigcup_{j=1}^{p}\left[c_{j}-\varepsilon, d_{j}+\varepsilon\right] .
\end{aligned}
$$

But $\bigcup_{j=1}^{p}\left[c_{j}-\varepsilon, d_{j}+\varepsilon\right] \cap D=\emptyset$, by the choice of $\varepsilon$. Since $H \subseteq D$, we have

$$
\left[y-b_{n}, y-a_{n}\right] \cap H=\left[y+a_{n}, y+b_{n}\right] \cap H=\emptyset
$$

which implies that $H \in R(\Phi, n) \cap \mathbb{K}$.

Note that the result analogous to Theorem 2 for strongly porous sets was obtained in [L]. The version for strongly shell porous sets in a complete space $X$ was shown in [V, Th. 2.1]. If $X=\mathbb{R}$, strong shell porosity coincides with strong symmetric porosity. 
It is known that the family Perf of all perfect subsets of $[0,1]$ is a $G_{\delta}$ subset of $\mathbb{K}[\mathrm{K}, \S 42$, III, Th. 3]. Hence Perf is a Polish space, by the Alexandrov theorem $[\mathrm{K}, \S 33, \mathrm{VI}]$. Now we establish a result which follows from Theorems 1 and 2 .

Theorem 3 The perfect $(\Phi)$-uniformly symmetrically porous sets in $[0,1]$ form $a G_{\delta}$ dense subset of Perf, and therefore it is a residual set in Perf.

Proof. Since $R(\Phi) \cap \mathbb{K}$ is a $G_{\delta}$ set in $\mathbb{K}$ (by Theorem 2), therefore $R(\Phi) \cap$ Perf is a $G_{\delta}$ set in Perf. It suffices to show that $R(\Phi) \cap$ Perf is dense in Perf. We will utilize the fact that the topology in $\mathbb{K}$ can equivalently be generated by the base consisting of sets of the form

$$
U\left(J_{0}, J_{1}, \ldots, J_{m}\right)=\left\{F \in \mathbb{K}:\left(F \subseteq J_{0}\right) \&(\forall j \in\{1, \ldots, m\})\left(F \cap J_{j} \neq \emptyset\right)\right\}
$$

where $J_{j}$, for $j=0,1, \ldots, m$, are open in $[0,1]$ (see $[\mathrm{K}, \S 42, \mathrm{II}]$ ). So, we will find a set from $R(\Phi) \cap$ Perf in a nonempty set $U\left(J_{0}, J_{1}, \ldots, J_{m}\right)$. We can assume that $J_{1} \cup \ldots \cup J_{m} \subseteq J_{0}$ and that $J_{1}, \ldots, J_{m}$ are pairwise disjoint (if the last condition is not fulfilled, we choose distinct points $x_{j} \in J_{j}$ for $j=1, \ldots, m$ and pairwise disjoint intervals $J_{j}^{\star} \subseteq J_{j}$ with $\left.x_{j} \in J_{j}^{\star}\right)$. Now, for each $j \in\{1, \ldots, m\}$, choose a perfect set $P_{j} \in R(\Phi), P_{j} \subseteq J_{j}^{\star}$ (we can use Theorem 1) and put $P=\bigcup_{j=1}^{m} P_{j}$. Then $P \in U\left(J_{0}, J_{1}, \ldots, J_{m}\right)$. Additionally, $P \in R(\Phi)$ (here we use the pairwise disjointness of $J_{1}, \ldots, J_{m}$ ) and obviously, $P \in$ Perf.

\section{References}

[B] M. Balcerzak, Some properties of ideals of sets in Polish spaces, Łódź University Press, 1991.

[H] M. Hejný, Typical intersections of continuous functions with monotone functions, Proc. Amer. Math. Soc., 118 (1993), 1131-1137.

[K] K. Kuratowski, Topology, vols I-II, PWN-Academic Press, WarszawaNew York 1966, 1968.

[L] L. Larson, Typical compact sets in the Hausdorff metric are porous, Real Analysis Exchange, 13 (1987-88), 116-118.

[V] R. W. Vallin, An introduction to shell porosity, Real Analysis Exchange, 18 (1992-93), 294-320.

[Z] L. Zajíček, Porosity and $\sigma$-porosity, Real Analysis Exchange, 13 (1987-88), 314-350. 